\title{
LXXIV. On some points relating to the theory of fluid motion
}

\author{
Rev. J. Challis M.A. F.R.S. F.R.A.S.
}

To cite this article: Rev. J. Challis M.A. F.R.S. F.R.A.S. (1849) LXXIV. On some points relating to the theory of fluid motion, Philosophical Magazine Series 3, 34:232, 512-520, DOI: 10.1080/14786444908646284

To link to this article: http://dx.doi.org/10.1080/14786444908646284

册 Published online: 30 Apr 2009.

Submit your article to this journal $\sqsubset \pi$

Џ Article views: 2

Q View related articles $\asymp$ 
LXXIV. On some Points relating to the Theory of Fluid Motion. By the Rev. J. Challis, M.A., F.R.S., F.R.A.S., Plumian Professor of Astronomy and Experimental Philosophy in the University of Cambridge*.

$\mathrm{N}$ a memoir on certain questions in the theory of the motion of fluids, published (1847) by Professor P. Tardy of Florence, for a copy of which $I$ am indebted to the author, reference is made to a communication contained in tom. xxiii. of the Comptes Rendus of the Academy of Sciences of Paris, in which several of my investigations on fluid motion are brought under review by M. J. Bertrand. Professor Tardy states at the same time, that he had himself previously made remarks on one of the points on which I am supposed to be in error. The citation of Professor Tardy first made me aware that M. Bertrand had taken notice of my labours. On turning to the article in the Comptes Rendus, I perceived that the important errors (erreurs graves) attributed to me were partly due to misconception of my reasoning, which, I am ready to admit, may not have been developed with sufficient clearness; and partly to the circumstance, not unusual in the history of science, that new truths appear to be errors so long as the errors they replace are supposed to be truths. It will suffice for the present to advert to one point of primary importance. I have repeatedly contended that, to complete the analytical theory of hydrodynamics, a new general equation is absolutely required. M. Bertrand first calls in question the principles on which this equation is established, and then contends that an equation which I derived from a combination of the new equation with that which is usually called the equation of continuity, is identical with a particular case of this latter equation. The following investigations will supply answers to these objections.

1. I propose first to exhibit the principles on which the new equation rests, and to deduce it accordingly. If the equation $\psi(x, y, z, t)=0$ express any given relation between the coordinates $x, y, z$ and the time $t$, any other relation between the same quantities may be expressed by the equation $\psi(x+\delta x, y+\delta y, z+\delta z, t+\delta t)=0$, the increments $\delta x, \delta y, \delta z$ being in general functions of the coordinates and the time. Supposing the increments to be indefinitely small, we obtain

$$
\frac{d \psi}{d t} \delta t+\frac{d \psi}{d x} \delta x+\frac{d \psi}{d y} \delta y+\frac{d \psi}{d z} \delta z=0
$$

* Communicated by the Author. 
If $u, v, w$ be the resolved parts of the velocity of a given particle of fluid in motion, and we suppose that

the above equation becomes

$$
\delta x=u \delta t \quad \delta y=v \delta t \quad \delta z=r o \delta t \text {, }
$$

$$
\frac{d \psi}{d t}+\frac{d \psi}{d x} u+\frac{d \psi}{d y} v+\frac{d \psi}{d z} w=0
$$

The signification of this equation depends entirely on the nature of the curve surface defined by the equation $\psi(x, y, z, t)=0$. If, for instance, this be the surface of a fixed or moveable boundary with which the fluid is in contact, the equation affirms that the same particle remains in contact with the boundary in successive instants. If the function $\psi$ be the general expression for the pressure $p$, then since $p=0$ is the equation of the free boundary, the above equation would express in this instance the condition that a given particle is situated on the free boundary in successive instants. Let now

$$
(d \psi)=\frac{u}{\lambda} d x+\frac{v}{\lambda} d y+\frac{w}{\lambda} d z \text {. }
$$

Then, as is known, $\psi=0$ is the equation of a surface cutting at right angles the directions of the motions of the particles through which it passes. The factor $\frac{1}{\lambda}$ is applied for the sake of generality, because it may be assumed that such a surface always exists, and consequently that the right-hand side of the above equality is an exact differential, although it cannot be affirmed that $u d x+v d y+w d z$ is always an exact differential. Assuming, therefore, the integrability of

it follows that

$$
\frac{u}{\lambda} d x+\frac{v}{\lambda} d y+\frac{w}{\lambda} d z
$$

$$
u=\lambda \frac{d \psi}{d x} . . \text { (1.) } \quad v=\lambda \frac{d \psi}{d y} . . \text { (2.) } \quad w=\lambda \frac{d \psi}{d z} \text {.. (3.). }
$$

Hence, substituting in the foregoing equation,

$$
\frac{d \psi}{d t}+\lambda\left(\frac{d \psi^{2}}{d x^{2}}+\frac{d \psi^{2}}{d y^{2}}+\frac{d \psi^{2}}{d z^{2}}\right)=0 . \text {. . . . }
$$

This is the new equation which it was proposed to obtain. The course of the investigation shows that this equation expresses the condition that the directions of the motion in a given element are in successive instants normals to surfaces of continued curvature. The fulfilment of this condition ensures the continuity of the motion; and the above equation may Phil. Mag. S. 3. No.232. Suppl. Vol. 34. 
consequently be called the equation of continuity, while the equation usually so named may with more propriety be called the equation of constancy of mass, with reference to the principle on which it is based.

To the equations (1.), (2.), (3.), and (4.) are to be added the two following:

$$
\begin{aligned}
& \frac{d \rho}{d t}+\frac{d \cdot \rho u}{d x}+\frac{d \cdot \rho v}{d y}+\frac{d \cdot \rho w}{d z}=0 \ldots \ldots \ldots \ldots \ldots \\
& \frac{(d p)}{\rho}+\left(\frac{d u}{d t}\right) d x+\left(\frac{d v}{d t}\right) d y+\left(\frac{d w}{d t}\right) d z=0 \ldots \ldots
\end{aligned}
$$

the fluid being supposed to be acted upon by no impressed forces. When the relation between the pressure $p$ and density $\rho$ is given, these six equations serve to determine the six unknown quantities, $\psi, \lambda, u, v, w$ and $\rho$.

The equation (5.) is equivalent to the following :

$$
\frac{d \rho}{d t}+\frac{d \rho}{d x} \cdot \frac{d x}{d t}+\frac{d \rho}{d y} \cdot \frac{d y}{d t}+\frac{d \rho}{d z} \cdot \frac{d z}{d t}+\rho\left(\frac{d u}{d x}+\frac{d v}{d y}+\frac{d r w}{d z}\right)=0 .
$$

Hence, if $u^{2}+v^{2}+w^{2}=\mathrm{V}^{2}$ and $d s=\mathrm{V} d t$,

$$
\frac{d \rho}{d t}+\mathrm{V} \cdot \frac{d \rho}{d s}+\rho\left(\frac{d u}{d x}+\frac{d v}{d y}+\frac{d w}{d z}\right)=0 \text {. }
$$

But by what is proved in the Cambridge Philosophical Transactions (vol. vii. part 3. p. 385, 386), where, however, it is proper to remark, the use of equation (4.) is not absolutely necessary, we have

$$
\frac{d u}{d x}+\frac{d v}{d y}+\frac{d w}{d z}=\frac{d \mathrm{~V}}{d s}+\mathrm{V}\left(\frac{1}{r}+\frac{1}{r^{1}}\right)
$$

$r$ and $r^{1}$ being the principal radii of curvature at the point $x y z$, of the surface which cuts the directions of motion at right angles. Hence, by substitution,

$$
\frac{d \rho}{d t}+\frac{d . V_{\rho}}{d s}+V_{\rho}\left(\frac{1}{r}+\frac{1}{r^{1}}\right)=0 \ldots \ldots
$$

This equation has also been derived from elementary considerations in the memoir above cited (p. 387). By whatever process it be obtained, it involves the principle expressed analytically by the equation (4.), viz. that the directions of motion are normals to surfaces of continued curvature. It cannot, therefore, be identical with any equation which does not involve the same principle, or does not contain explicitly the radii of curvature $r$ and $r^{1}$. An application of the equation 
will illustrate this remark. Suppose the fluid to be incompressible: then

$$
\frac{d \mathrm{~V}}{d s}+\mathrm{V}\left(\frac{1}{r}+\frac{1}{r^{1}}\right)=0 \text {. }
$$

Hence, since $d s=d r=d r^{1}$, we have by integrating,

$$
\mathrm{V}=\frac{\phi(t)}{r r^{1}}
$$

This expression for the velocity is general, having been obtained prior to the consideration of any particular case of motion. It establishes the general law, that the quantity of fluid which passes in a given small time a given small element of a surface of displacement, being proportional to $\mathrm{Vrr}^{1}$, is given for a given value of $\phi(t)$, and consequently that the momentary trajectory of the surfaces of displacement to which a single disturbance gives rise is a straight line.

If the motion take place in space of two dimensions, we have

$$
\mathrm{V}=\frac{\phi(t)}{r}
$$

I formerly obtained this result for the case in which $u d x+$ $v d y$ is an exact differential, by a process which Professor Tardy and M. Bertrand object to, and which I do not now insist upon, because the above reasoning is inclusive of the particular case, and the result is obtained in a more direct manner.

To apply the above general value of $\mathrm{V}$ to a given instance: suppose a perfectly smooth sphere to move in any manner in an incompressible fluid of unlimited extent, its centre remaining on a given straight line. The general value of $\mathrm{V}$ applies to the velocity impressed on the fluid by the surface of the sphere. If $V^{\prime}$ be the velocity of its centre, then the motion impressed at any point of the surface the radius to which makes an angle $\theta$ with the direction of motion, is $V^{1} \cos \theta$. Hence, $R$ being the radius of the sphere,

$$
V^{1} \cos \theta=\frac{\phi(t)}{R^{2}}, \text { or } \phi(t)=R^{2} V^{1} \cos \theta \text {. }
$$

Consequently, as there is no other arbitrary quantity to satisfy,

$$
\mathrm{V}=\frac{\mathrm{R}^{2}}{r^{2}} \mathrm{~V}^{1} \cos \theta
$$

where $r^{2}$ is substituted for $r^{1}$, because as one surface of dis placement is spherical, all are spherical, their momentary trajectory being rectilinear. This result does not agree with that 2 L 2 
given by the received hydrodynamical equations employed in the usual manner, - first, because those equations do not include the principle of continuity expressed by equation (4.); and secondly, because in treating this problem, $u d x+v d y+w d z$ has without reason been assumed to be an exact differential.

2. I proceed next to trace the consequences of introducing into the general equations the condition that $u d x+v d y+w d z$ is an exact differential. Let $(d \phi)=u d x+v d y+w d z$. Then $(d \phi)=0$ is the differential of the equation of a surface cutting at right angles the directions of motion. Hence the value of $\frac{1}{r}+\frac{1}{r^{t}}$ in equation (7.) may be expressed by means of the

partial differential coefficients of $\phi$. This expression being substituted in (7.), and $\rho$ being eliminated by means of (5.) and (6.), the result is identical with the known equation $(n)$ in the Mécanique Analytique (Part 2. Section XII. p. 344). I have indicated this process for the purpose of remarking, that it does not thence follow that equations (7.) and $(n)$ are identical, or that the former is a particular case of the latter. Both equations are equally general. The essential difference between them is, that (7.) involves an expression of the condition that the directions of motion are normals to surfaces of continued curvature; whereas $(n)$ involves no expression of this condition, being usually obtained by simply supposing $\phi$ to be a certain function, the partial differential coefficients of which with respect to $x, y$ and $z$ are respectively $u, v$ and $w$. It is not possible to pass from $(n)$ to (7.) without introducing this condition of continuity, and equation (7.) consequently signifies something more than equation $(n)$.

We have now to consider the change which equation (4.) undergoes by supposing $u a^{3} x+v d y+r o d z$ to be an exact differential. It might at first sight be supposed that for this purpose it is sufficient to put unity for $\lambda$. That this would be a false step is clear from the consideration, that there would then be six equations and but five unknown quantities, without any reasons for concluding that the equations would be consistent with each other. In fact, it would be found on trial that on this supposition they are inconsistent. The only legitimate process is to trace the consequence of supposing $u d x+v d y+r o d z$ to be an exact differential, by reasoning generally according to the rules of analysis.

Between the functions $\phi$ and $\psi$ we have the relation, that $(d \psi)=0$ and $(d \phi)=0$ are both differential equations of the same curve surface. But $(d \phi)=0$ being the differential equation of a curve surface, it is clear that $(d . F(\varphi))=0$, or $F^{\prime}(\phi)(d \phi)=0$, 
is a differential equation of the same surface. Consequently

But

$$
(d \psi)=\mathrm{F}^{\prime}(\phi)(d \varphi) \text {. }
$$

therefore

$$
(d \psi)=\frac{1}{\lambda}(d \phi) ;
$$

Also by integration,

$$
\lambda=\frac{1}{\mathbf{F}^{\prime}(\phi)}
$$

Hence

$$
\psi=\mathrm{F}(\varphi)+\chi(t)
$$

$$
\begin{array}{ll}
\frac{d \psi}{d t}=\mathrm{F}^{\prime}(\phi) \frac{d \phi}{d t}+\chi^{\prime}(t) & \frac{d \psi}{d x}=\mathrm{F}^{\prime}(\phi) \frac{d \phi}{d x} \\
\frac{d \psi}{d y}=\mathrm{F}^{\prime}(\phi) \frac{d \phi}{d y} & \frac{d \psi}{d z}=\mathrm{F}^{\prime}(\phi) \frac{d \phi}{d z} .
\end{array}
$$

Consequently by substituting in (4.), and having regard to the value of $\lambda$, we obtain

$$
\frac{d \phi}{d t}+\frac{d \phi^{2}}{d x^{2}}+\frac{d \phi^{2}}{d y^{2}}+\frac{d \phi^{2}}{d z^{2}}+\frac{\chi^{\prime}(t)}{\mathrm{F}^{\prime}(\phi)}=0 . \quad .
$$

Thus we have an equation involving the same variables as the general equation $(n)$ already referred to, and yet not identical with it. The interpretation of this analytical circumstance is, that the function $\phi$ has not an arbitrary, but a particular form; and it is a matter of importance to ascertain what that form is. The following investigation may perhaps suffice for this purpose, but is not the most general that might be adopted. For the sake of avoiding long processes I shall confine the reasoning to the first order of approximation.

The equation (8.) may be put under the form,

$$
\frac{d \varphi}{d t}+\frac{d \phi^{2}}{d s^{2}}+\frac{\chi^{\prime}(t)}{\mathbf{F}^{\prime}(\varphi)}=0, \quad \text {. . . . }
$$

where $d s$ is the increment of a line drawn in the direction of the motion, so that $\frac{d \phi}{d s}=\mathrm{V}$. Now neglecting the term involving $V^{2}$, we have

$$
\left.\mathbf{F}^{\prime} \varphi\right) \frac{d \phi}{d t}+\chi^{\prime}(t)=0
$$

and by integration,

Hence

$$
\mathrm{F}(\phi)+\chi(t)=\theta(s)
$$

$$
\phi=f(\theta(s)-\chi(t)) \text {. }
$$


But we have already found, without using equation (9.), that

Hence

$$
\mathbf{F}(\phi)+\chi(t)=\psi \text {. }
$$

$$
\varphi=f(\psi-\chi(t)) \text {. }
$$

A comparison between these two values of $\phi$ gives

$$
\psi=\theta(s) \text {. }
$$

The above value of $\phi$ must satisfy the following linear equation:

$$
0=a^{2}\left(\frac{d^{2} \varphi}{d x^{2}}+\frac{d^{2} \varphi}{d y^{2}}+\frac{d^{2} \phi}{d z^{2}}\right)-\frac{d^{2} \phi}{d t^{2}} . \quad .
$$

It may therefore be assumed that $\theta(s)$ and $\chi(t)$ are linear quantities, and accordingly that

$$
\theta(s)=s \text {, and } \chi(t)=c t .
$$

Also since $\psi=0$ is the equation of a curve surface, we have in general,

Hence

$$
\psi=z+c^{\prime}+q(x, y, t) \text {. }
$$

$$
s=z+c^{\prime}+q(x, y, t) \text {. }
$$

It does not appear possible to satisfy this last condition unless the line of motion be rectilinear; that is, unless the motion be along an axis, which may be supposed to coincide with the axis of $z$. 'The function $q$, being of given form depending on the equation of the surface, cannot express the value of $s$. This function must therefore disappear on making $x=0$ and $y=0$; and we thus obtain

and

$$
s=z+c^{\prime},
$$

$$
\varphi=f\left(z+c^{\prime}-c t\right) \text {. }
$$

This value applies strictly to motion along the axis of $z$. Before proceeding to substitute in equation (10.), it is necessary to express the value of $\varphi$ for points immediately contiguous to the axis. For this purpose suppose

$$
\phi=f\left(z+c^{\prime}+q(x, y, t)-c t\right) .
$$

Then substituting the letters $f$ and $q$ for the functions themselves, we shall have

$$
\begin{gathered}
\frac{d^{2} \phi}{d x^{2}}=f^{\prime \prime} \cdot \frac{d q^{2}}{d x^{2}}+f^{\prime} \frac{d^{2} q}{d x^{2}}=f^{\prime} \cdot \frac{d^{2} q}{d x^{2}}, \quad \text { because } \frac{d q}{d x}=0, \\
\frac{d^{2} \varphi}{d y^{2}}=f^{\prime} \cdot \frac{d^{2} q}{d y^{2}}, \quad \frac{d^{2} \varphi}{d z^{2}}=f^{\prime \prime}, \quad \frac{d^{2} \varphi}{d t^{2}}=f^{\prime \prime}\left(\frac{d q}{d t}-c\right)^{2}+f^{\prime} \frac{d^{2} q}{d t^{2}} .
\end{gathered}
$$


Al so supposing that

it follows that

$$
q=\alpha x+\beta y+g x^{2}+h x y+k y^{2}+\& c \text {., }
$$

$$
\begin{aligned}
& \frac{d q}{d x}=\alpha=0, \quad \frac{d q}{d y}=\beta=0, \quad \frac{d^{2} q}{d x^{2}}=2 g, \\
& \frac{d^{2} q}{d y^{2}}=2 k, \quad \frac{d q}{d t}=0, \text { and } \frac{d^{2} q}{d t^{2}}=0 .
\end{aligned}
$$

The coefficient $h$ may be made to disappear by changing the direction of co-ordinates, and $g$ and $k$ must be supposed to be independent of the time. Hence by substituting in (10.) we obtain for determining the form of $f$ the following equation :

$$
f^{\prime \prime}-\frac{2(g+k) a^{2}}{c^{2}-a^{2}} f^{\prime}=0 \text {. }
$$

Putting $m$ for the coefficient of $f^{\prime}$, and $\nu$ for the quantity of which $f$ is a function, the integral of this equation becomes

$$
f=\mathrm{A} e^{m y}+\mathrm{B} \text {. }
$$

The form of $f$ is thus ascertained. Since $q$ is of arbitrary value, we may multiply it by $\sqrt{-1}$, and the value of $f$ will then become

$$
f=\mathrm{A} e^{-\left(\mathrm{G} x^{2}+\mathrm{H} y^{2}\right)} \cdot e^{m\left(z+c^{\prime}-c t\right) \sqrt{ }-\overline{1}}+\mathrm{B} .
$$

The equation (10.) being linear, may be satisfied by the sum of this value and that which results by changing the sign of $\sqrt{-1}$. So that putting $x=0$ and $y=0$, and suppressing the constant $B$, the final result is,

$$
\varphi=\mu \cos m\left(z+c^{\prime}-c t\right) \text {. }
$$

By putting $\frac{2 \pi}{\lambda}$ for $m$, the resulting value of $c$ is the following,

$$
c=a\left(1+\frac{(g+k) \lambda}{\pi}\right)^{\frac{1}{2}} .
$$

This value agrees with what $I$ have previously obtained in a different manner. The reasoning in the present method is more direct, in consequence of the use that has been made of the new hydrodynamical equation. I shall conclude this investigation with the remark, that the results arrived at are wholly incompatible with those deduced from the supposition of plane-waves, although the reasoning proceeded on the general hypothesis that $u d x+v d y+$ rodz was an exact differential, and ought not, if that supposition were allowable, to have led to 
any contradiction. I infer that the supposition of plane-waves is not allowable.

I have been induced to make the last remark from having seen it asserted by the Astronomer Royal in the Number of the Philosophical Magazine for June, that I have pointed out a difficulty in the interpretation of an equation applying to the case of plane-waves. Mr. Stokes asserted the same thing before; and I then disclaimed, as I now disclaim, having pointed out any difficulty. The equation is a very simple one, and easily interpreted. A few steps of plain deduction conducts to a result incompatible with fluid motion. It follows in due course that the supposition of plane-waves cannot be made. This inference is in perfect accordance with the argument contained in this communication, which I think Mr. Airy may find to be worthy of some consideration. Any other inference would have presented a real difficulty.

Cambridge Observatory, June 22, 1849.

LXXV. Appendix to Mr. Drach's Paper on Epicyclic Curves in the last June Number.

A NOTHER example, with series expanded.

$$
n=-\frac{5}{2} \therefore p+q=7, \quad q=2 .
$$

Let $\mathbf{Q}=\mathbf{Q}^{\prime} \cdot a b$. The equation is

$$
\begin{aligned}
2 x^{7}- & 7 r^{2} \cdot(2 x)^{5}+14 r^{4}(2 x)^{3}-7 r^{6}(2 x)=a^{7}\left(\mathbf{Q}^{\prime 2}-2\right)+7 a^{6} b \mathbf{Q}^{\prime} \\
& +42 a^{5} b^{2}+35 a^{4} b^{3} \mathbf{Q}^{\prime}+35 a^{3} b^{4}\left(\mathbf{Q}^{\prime 2}-2\right)+21 a^{2} b^{5}\left(\mathbf{Q}^{\prime 3}-3 \mathbf{Q}^{\prime}\right) \\
& +7 a b^{6}\left(\mathbf{Q}^{\prime 4}-4 \mathbf{Q}^{\prime 2}+2\right)+b^{7}\left(\mathbf{Q}^{\prime 5}-5 \mathbf{Q}^{\prime 3}+5 \mathbf{Q}^{\prime}\right) .
\end{aligned}
$$

When $a=b$, the second member

$$
\begin{aligned}
= & a^{7}\left\{\left(Q^{\prime}+2\right)^{5}-3\left(Q^{\prime}+2\right)^{4}=r^{10} a^{-10}-3 r^{8} a^{-8}\right\} \\
& =r^{7}\left\{r^{3} a^{-3}-3 r a^{-1}\right\},
\end{aligned}
$$

agreeably to Case 2 .

The following errors have to be corrected in the last Number.

Ex.

$$
n=-3 \text { for } \frac{\mathbf{Q}}{a^{3} b} \operatorname{read} \frac{\mathbf{Q} a^{3}}{b}
$$

